\section{Mortalidade perinatal no Brasil em 2018: análise epidemiológica segundo a classificação de Wiggleworth modificada}

\author{
Perinatal mortality in Brazil in 2018: an \\ epidemiological analysis according to the \\ modified Wigglesworth classification
}

\section{Mortalidad perinatal en Brasil en 2018: análisis epidemiológico según la clasificación de Wiggleworth modificada}

Aglaer Alves da Nobrega 1 Yluska Myrna Meneses Brandão e Mendes 1 Marina Jorge de Miranda 1 Augusto César Cardoso dos Santos 1 Andréa de Paula Lobo 1

Denise Lopes Porto 1

Giovanny Vinícius Araújo de França 1

\title{
Resumo
}

A mortalidade perinatal engloba a mortalidade fetal e a neonatal precoce $(0 \mathrm{a}$ 6 dias). Este estudo descreveu os óbitos perinatais ocorridos no Brasil em 2018, segundo a classificação de Wigglesworth modificada. As fontes de dados foram os Sistemas de Informações sobre Mortalidade e sobre Nascidos Vivos. Foram calculadas as taxas de mortalidade fetal e perinatal por mil nascimentos totais (nascidos vivos mais natimortos) e a taxa de mortalidade neonatal precoce por mil nascidos vivos, e comparadas usando seus respectivos intervalos de 95\% de confiança (IC95\%). Os óbitos perinatais foram classificados nos grupos de causas anteparto, anomalias congênitas, prematuridade, asfixia e causas específicas. Foi calculado, para cada grupo de causas, o número de óbitos por faixa de peso, além das taxas de mortalidade e os respectivos IC95\%, e feita a distribuição espacial das taxas de mortalidade por Unidade da Federação (UF). Foram registrados 35.857 óbitos infantis, sendo 18.866 (52,6\%) neonatais precoces; os natimortos somaram 27.009. Os óbitos perinatais totalizaram 45.875, perfazendo uma taxa de mortalidade de $15,5 \%$ nascimentos. A maior taxa de mortalidade (7,6\%; 7,5\%-7,7\%) foi observada no grupo anteparto, seguido da prematuridade (3,6\%o; 3,6\%o-3,7\%o). No grupo anteparto, 14 das 27 UFs (sendo oito na Região Nordeste e quatro na Região Norte) apresentaram as taxas de mortalidade perinatal acima da nacional. A taxa de mortalidade perinatal no Brasil mostrou-se elevada, e a maioria dos óbitos poderia ser prevenida com investimento em cuidados pré-natais e ao nascimento.

Mortalidade Perinatal; Óbito Fetal; Morte Neonatal

Correspondência

A. A. Nobrega

Secretaria de Vigilância em Saúde, Ministério da Saúde. SRTVN 701, Via W5 Norte, Edifício PO700, 6o andar, Brasília, DF 70723-040, Brasil.

aglaeran@yahoo.com.br

1 Secretaria de Vigilância em Saúde, Ministério da Saúde, Brasília, Brasil. 


\section{Introdução}

A mortalidade perinatal é compreendida como os óbitos ocorridos entre a 22a semana de gestação e o sexto dia completo de vida após o nascimento. Ou seja, engloba a mortalidade fetal e a neonatal precoce ( 0 a 6 dias) 1. A análise conjunta dessas mortalidades é importante porque as causas de mortes fetais e neonatais precoces são similares e, frequentemente, relacionadas às condições do pré-natal, do parto e do recém-nascido. Assim, as intervenções que reduzem a natimortalidade e a mortalidade neonatal precoce também reduzem a mortalidade materna 2,3,4,5,6.

Os óbitos perinatais são eventos potencialmente evitáveis e refletem a qualidade da assistência prestada no pré-natal, no parto e nos primeiros dias de vida do recém-nascido 7,8. Com o declínio da mortalidade na infância, a mortalidade perinatal emergiu como um problema de saúde pública, sobretudo nos países de baixa e média renda 9 . Apesar de observada uma redução de óbitos fetais no mundo, as mortes neste grupo, bem como a dos recém-nascidos, estão reduzindo mais lentamente que aquelas ocorridas em menores de cinco anos e mortes maternas 10.

Nesse contexto, ressalta-se que, globalmente, as mortes de recém-nascidos correspondem a $44 \%$ do total de óbitos de menores de cinco anos 10. Sobre os conceptos que nasceram mortos, estimase que, em 2015, foram 2,6 milhões, podendo variar entre 2,4 e 3 milhões. Daquele total, 98\% das mortes ocorreram em países de baixa e média renda, sendo $77 \%$ concentrados no sul da Ásia e na África Subsaariana 9.

No Brasil, a taxa de mortalidade infantil caiu $80,4 \%$ nas últimas décadas, passando de 71,3 por mil nascidos vivos, em 1982, a 14, em 2015. Já a mortalidade neonatal reduziu 63,4\%, e passou de 33,4 para 8,2 por mil no mesmo período, uma redução $17 \%$ menor quando comparada à mortalidade infantil. Entre as mortes neonatais, o declínio foi mais lento na mortalidade neonatal precoce do que na mortalidade neonatal tardia (7 a 28 dias) 11. A taxa de mortalidade fetal passou de 8,19, em 1996, para 9,5/1.000 nascimentos, em 2015 e, desde 2000, apresenta um quadro estacionário no Brasil e em todas as regiões 12 .

Dado o desafio que é reduzir a mortalidade perinatal 13, estudos nacionais sobre esse tema ainda se fazem necessários e podem ser úteis para evidenciar a qualidade dos serviços de saúde e sugerir medidas para reduzir os óbitos perinatais.

O objetivo desse estudo foi descrever e classificar os óbitos perinatais ocorridos no Brasil, segundo a classificação de Wigglesworth modificada, no ano de 2018.

\section{Método}

Foi realizado um estudo descritivo dos óbitos perinatais ocorridos no Brasil em 2018. A Organização Mundial da Saúde (OMS) define óbito fetal como a morte de um produto da concepção, antes da expulsão ou da extração completa do corpo da mãe, independentemente da duração da gravidez. O óbito é constatado quando, após a separação, não houver respiração ou outro sinal de vida, como batimentos do coração, pulsação do cordão umbilical ou movimentos efetivos dos músculos de contração voluntária. O nascido vivo é o produto da expulsão ou extração completa do corpo da mãe, independentemente da duração da gestação, de um produto de concepção que, depois da separação, respire ou apresente qualquer sinal de vida como batimentos do coração, pulsação do cordão umbilical ou movimentos efetivos dos músculos de contração voluntária, estando ou não cortado o cordão umbilical e estando ou não desprendida a placenta 1.

Para o período perinatal, adotou-se a definição utilizada pelo Ministério da Saúde: inicia-se em 22 semanas completas de gestação e termina aos sete dias completos de vida. Desta forma, para o estudo, óbito perinatal é aquele ocorrido nesse período 14 .

As fontes de dados foram os Sistemas de Informações sobre Mortalidade (SIM) e o Sistema de Informações sobre Nascidos Vivos (SINASC), sistemas oficiais de captação de óbitos e nascimentos vivos no Brasil, respectivamente ${ }^{15}$. Os dados contidos em ambos os sistemas servem de base para o cálculo de importantes indicadores de saúde nos níveis federal, estadual e municipal.

O SIM, implantado em 1975, tem como documento base e padronizado para captação de dados sobre óbito em todo o país a Declaração de Óbito (DO) 15. Em 2018, apresentava uma cobertura nacio- 
nal de 95,8\%, variando entre $89,1 \%$ e $100 \%$ nas Unidades de Federação (UFs). A cobertura nacional para o óbito infantil, em 2018 , foi de $91,1 \%$, variando de $78,2 \%$ a $100 \% 16$.

A implantação do SINASC nas UFs ocorreu no início de 1990, de forma gradual. O registro dos dados sobre nascimentos se dá por meio da Declaração de Nascido Vivo (DNV) 17. Em 2018, o sistema apresentava uma cobertura nacional de 97,6\%, variando entre $91 \%$ e $100 \%$ nas UFs 16 .

As taxas de mortalidade fetal e perinatal por mil nascimentos totais (nascidos vivos mais óbito fetal) e a taxa de mortalidade neonatal precoce por mil nascidos vivos foram calculadas pelo método direto e comparadas usando seus respectivos intervalos de 95\% de confiança (IC95\%). Para o cálculo da taxa de mortalidade fetal foi somado ao numerador e ao denominador o número de óbitos fetais com idade gestacional ignorada ou não preenchida 14,18. Assim, dos 27.009 óbitos fetais ocorridos em 2018, estavam com idade gestacional ignorada $2.338(8,7 \%)$.

As variáveis analisadas foram sexo (masculino e feminino); peso ao nascer, em gramas $(<500$; 500-999, 1.000-1.499, 1.500-2.499, 2.500 ou mais); idade materna, em anos (<20, 20-34, $\geq 35)$; escolaridade materna, em anos de estudo (0-3, 4-7, 8-11, 12 ou mais); idade gestacional, em semanas (<22; 22-27, 28-31, 32-36, 37-41, 42 ou mais); tipo de gravidez (única, múltipla) e tipo de parto (vaginal, cesáreo). Os nascimentos vivos com peso inferior a $500 \mathrm{~g}$ e idade gestacional inferior a 22 semanas foram incluídos apenas no cálculo da mortalidade neonatal precoce.

Os óbitos perinatais foram agrupados, segundo a classificação de Wigglesworth 19 modificada por Keeling et al. 20, em cinco grupos de causas excludentes: anteparto, anomalias congênitas, prematuridade, asfixia e causas específicas. Essa classificação é baseada em grupos com implicações claras para o manejo clínico (Quadro 1) 19,20.

O grupo anteparto foi composto por todos os óbitos fetais ocorridos antes do trabalho de parto. Para o grupo de anomalias congênitas, foram consideradas como malformação letal ou potencialmente letal aquelas descritas por Wilkinson et al. 21 (Q00-Q01, Q03, Q04.2-Q04.3, Q04.9, Q05,Q20-Q28, Q33.6, Q60.1-Q60.2, Q60.6, Q61.0-Q61.4, Q61.9, Q77.1,Q78.0, Q79.0,Q79.2-Q79.3, Q87.2, Q89.4, Q89.7-Q89.9, Q91, Q92.7). No grupo de prematuridade, foram incluídos os nascidos vivos com menos de 37 semanas de gestação e todos os nascidos vivos com peso ao nascer menor que 1.000g. Para avaliar trauma de parto e asfixia, foram incluídas as causas básicas do capítulo XVI da Classificação Internacional de Doenças - 10a revisão (CID-10) 1, classificando o traumatismo de parto

\section{Quadro 1}

Grupos de causas de óbito perinatal, segundo classificação de Wigglesworth modificada 20.

\begin{tabular}{|l|c|}
\hline \multicolumn{1}{|c|}{ GRUPOS } & \multicolumn{1}{|c|}{ TAXAS ELEVADAS } \\
\hline (1) Anteparto: morte fetal que ocorre antes do trabalho de parto & Falhas na atenção ao pré-natal e condições maternas adversas \\
\hline (2) Anomalias congênitas & $\begin{array}{c}\text { Falhas no diagnóstico e/ou terapia na gravidez } \\
\text { (lesões potencialmente tratáveis) }\end{array}$ \\
\hline $\begin{array}{l}\text { (3) Prematuridade: nascidos vivos com menos de 37 semanas de } \\
\text { gestação, sem hipóxia/anóxia; todos os nascidos vivos com peso ao } \\
\text { nascer menor que 1.000g }\end{array}$ & Falhas no manejo obstétrico e pré-natal/neonatal \\
\hline $\begin{array}{l}\text { (4) Asfixia: perda fetal intraparto; óbito fetal sem maceração; } \\
\text { natimorto recente (menos que 4h); óbitos neonatais por hipóxia, } \\
\text { exceto peso ao nascer menor que 1.000g }\end{array}$ & Falhas no manejo obstétrico e/ou reanimação neonatal \\
\hline $\begin{array}{l}\text { (5) Causas específicas: óbito por infecções especifica - STORCH *, e } \\
\text { óbito neonatal precoce, com 1.000g ou mais de peso e que foram a } \\
\text { óbito com 4h ou mais após o nascimento e com 37 ou mais semanas } \\
\text { de gestação }\end{array}$ & Falhas na assistência pré-natal e assistência ao recém-nascido \\
\hline
\end{tabular}

* Sífilis, toxoplasmose, rubéola, citomegalovírus, vírus herpes simplex. 
de P10 a P15; a asfixia, a hipóxia intrauterina (P20) e a asfixia ao nascer (P21). As causas específicas incluíram óbitos por sífilis, toxoplasmose, rubéola, citomegalovírus, vírus herpes simplex (STORCH), óbitos neonatais precoces, com $1.000 \mathrm{~g}$ ou mais de peso e que foram a óbito com $4 \mathrm{~h}$ ou mais após o nascimento e com 37 ou mais semanas de gestação. As causas básicas finais do óbito, confirmadas após investigação 22, foram informadas por meio de códigos da CID-10 1.

No estudo de Keeling et al. 20, utilizado como referencial teórico, os pesquisadores usaram dados de prontuários, clínicos, resultados de necropsias macroscópicas, histológicas, dentre outros. Todavia, de posse desses resultados, eles propuseram uma árvore de decisão, cujos pontos colocados são captados pelo SIM. Desta forma, foi possível aplicar a referida classificação e realizar as análises para o conjunto dos óbitos perinatais ocorridos no Brasil em 2018 por meio das variáveis do SIM. Da mesma forma, essa análise pode ser reproduzida com os dados estaduais, municipais e das regiões de saúde.

Foram calculadas, pelo método direto, as taxas de mortalidade por mil e os respectivos IC95\%, bem como o número de óbitos segundo a faixa de peso em cada grupo de causas. Os cálculos foram realizados pelo Microsoft Excel (https://products.office.com/). Para o cálculo das taxas, procedeu-se da seguinte forma nos grupos de causas de óbito:

(1) Anteparto: óbitos no anteparto divididos pela soma dos óbitos no anteparto com o total de nascidos vivos, multiplicado por mil;

(2) Anomalias congênitas: óbitos por anomalias divididos pela soma dos óbitos por anomalias com o total de nascidos vivos, multiplicado por mil;

(3) Prematuridade: óbitos por prematuridade (nascido vivo $<1.000 \mathrm{~g} \mathrm{e}<37$ semanas de gestação) divididos pela soma dos óbitos por prematuridade com o total de nascidos vivos, multiplicado por mil;

(4) Asfixia: óbitos por asfixia (intraparto + trauma $+<4$ h de vida) divididos pela soma dos óbitos por asfixia com o total de nascidos vivos, multiplicado por mil;

(5) Causas específicas: óbitos por causas específicas ( $\mathrm{STORCH} \mathrm{+} \mathrm{>} 37$ semanas de gestação), divididos pela soma dos óbitos por causas específicas com o total de nascidos vivos, multiplicado por mil.

Além disso, foi realizada a distribuição espacial das taxas de mortalidade entre as UFs, por cada grupo de causa, por UF. O software utilizado foi QGis, versão 2.18 (https://qgis.org/en/site/).

Os dados utilizados no estudo foram de bases secundárias e não nominais, disponibilizadas pelo Departamento de Análise em Saúde e Doenças não Transmissíveis da Secretaria de Vigilância em Saúde do Ministério da Saúde. Dessa forma, encontram-se em conformidade com o Decreto no 7.724/201223 e com a Resolução no 510/2016 24, que dispõem sobre o acesso a informações e sobre as normas aplicáveis a pesquisas, respectivamente.

\section{Resultados}

Em 2018, foram registrados 35.857 óbitos infantis: 18.866 (52,6\%) neonatais precoces; os óbitos fetais somaram 27.009, representando $58,9 \%$ dos óbitos perinatais. Assim, os óbitos perinatais totalizaram 45.875, perfazendo uma taxa de mortalidade de 15,5\% nascimentos totais (Tabela 1).

As maiores taxas de mortalidade perinatal, a cada mil nascimentos, foram observadas nos óbitos do sexo masculino (16,2\%; $16 \%$ - $16,4 \%$ ) e de peso entre $500 \mathrm{~g}$ e $999 \mathrm{~g}(602,4 \%$; $595,7 \%$ - $609 \%$ ). As mães com idade maior que $34 \mathrm{e}$ menor que 20 anos apresentaram as maiores taxas sendo, respectivamente, $17,7 \%_{0}\left(17,3 \%_{0}-18 \%\right.$ ) e $16,4 \%$ o (16\% - $16,7 \%$ o) nascimentos. A maior taxa (50,6\%; 48,9\%o-52,2\%o) foi registrada entre os conceptos das mulheres com baixa escolaridade (Tabela 1).

Os óbitos perinatais ocorridos entre a 32a e 36a semanas de gestação mostraram uma taxa de mortalidade, por mil nascimentos totais, quase nove vezes maior que aquela observada no período gestacional seguinte. A gravidez múltipla $(51,9 \%$; $50,2 \%$ - $53,6 \%$ ) e o parto vaginal $(20,5 \%$; $20,3 \%$ 20,8\%) também apresentaram as maiores taxas (Tabela 1).

Quando os óbitos perinatais foram distribuídos seguindo a classificação de Wigglesworth modificada 20 , a maioria $(22.667 ; 51,8 \%)$ ocorreu antes do parto e tinham menos de 1.000 g; e $4.714(10,8 \%)$ eram portadores de anomalia congênita letal ou potencialmente letal (Figura 1). A prematuridade totalizou $10.704(24,5 \%)$, asfixia, $3.281(7,5 \%)$ e as causas especificas, $2.384(5,4 \%)$. Dos 558 registros de STORCH, 551 (98,7\%) foram por sífilis congênita. 
Tabela 1

Número de óbitos e taxa de mortalidade fetal, neonatal precoce e perinatal. Brasil, 2018.

\begin{tabular}{|c|c|c|c|c|c|c|}
\hline \multirow[t]{2}{*}{ Variáveis } & \multicolumn{2}{|c|}{ Óbito fetal * } & \multicolumn{2}{|c|}{ Óbito neonatal precoce ** } & \multicolumn{2}{|c|}{ Óbito perinatal * } \\
\hline & $\mathbf{n}$ & Taxa (IC95\%) & $\mathbf{n}$ & Taxa (IC95\%) & $\mathbf{n}$ & Taxa (IC95\%) \\
\hline Total & 27.009 & $9,1(9,0-9,2)$ & 18.866 & $6,4(6,3-6,5)$ & 45.875 & $15,4(15,3-15,6)$ \\
\hline \multicolumn{7}{|l|}{ Sexo } \\
\hline Masculino & 14.110 & $9,3(9,1-9,4)$ & 10.517 & $7(6,7-7,1)$ & 24.627 & $16,2(16,0-16,4)$ \\
\hline Feminino & 12.402 & $8,6(8,4-8,7)$ & 8.156 & $5,7(5,6-5,8)$ & 20.558 & $14,2(14,0-14,4)$ \\
\hline \multicolumn{7}{|l|}{ Peso (g) } \\
\hline$<500$ & - & - & 1.815 & $461,8(446,4-477,4)$ & - & - \\
\hline $500-999$ & 6.631 & $316,5(310,2-322,8)$ & 5.988 & $418,2(410,1-426,3)$ & 12.619 & $602,4(595,7-609)$ \\
\hline $1.000-1.499$ & 4.214 & $159,2(154,8-163,6)$ & 2.513 & $112,9(108,8-117,1)$ & 6.727 & $254,2(248,9-259,4)$ \\
\hline $1.500-2.499$ & 6.951 & $32,1(31,3-32,8)$ & 3.254 & $15,5(15,0-16,0)$ & 10.205 & $47,1(46,2-48,0)$ \\
\hline 2.500 ou mais & 7.273 & $2,7(2,6-2,8)$ & 4.224 & $1,6(1,5-1,6)$ & 11.497 & $4,3(4,2-4,3)$ \\
\hline \multicolumn{7}{|c|}{ Idade da mãe (anos) } \\
\hline$<20$ & 4.058 & $8,8(8,6-9,1)$ & 3.461 & $7,6(7,3-7,8)$ & 7.519 & $16,4(16,0-16,7)$ \\
\hline $20-34$ & 15.817 & $7,7(7,6-7,8)$ & 11.071 & $5,5(5,4-5,6)$ & 26.888 & $13,1(13,0-3,3)$ \\
\hline$\geq 35$ & 5.080 & $11(10,7-11,3)$ & 3.086 & $6,8(6,5-7,0)$ & 8.166 & $17,7(17,3-18,0)$ \\
\hline \multicolumn{7}{|c|}{ Escolaridade da mãe (anos de estudo) } \\
\hline $0-3$ & 2.286 & $32,7(31,3-34)$ & 1.253 & $18,5(17,5-19,5)$ & 3.539 & $50,6(48,9-52,2)$ \\
\hline $4-7$ & 5.406 & $12,1(11,8-12,4)$ & 3.408 & $7,7(7,5-8,0)$ & 8.814 & $19,8(19,4-20,2)$ \\
\hline $8-11$ & 11.657 & $6,5(6,4-6,6)$ & 8.994 & $5,1(4,9-5,2)$ & 20.651 & $11,5(11,4-11,7)$ \\
\hline 12 ou mais & 2.871 & $4,7(4,5-4,8)$ & 2.634 & $4,3(4,1-4,5)$ & 5.505 & $8,9(8,7-9,2)$ \\
\hline \multicolumn{7}{|c|}{ Idade gestacional (semanas) } \\
\hline$<22$ & - & - & 1.286 & $866,6(849,3-883,9)$ & - & - \\
\hline $22-7$ & 5.496 & $274(267,9-280,2)$ & 6.076 & $417,3(409,3-425,3)$ & 11.572 & $577,0(570,2-583,9)$ \\
\hline $28-31$ & 4.776 & $138,7(135,1-142,4)$ & 2.884 & $97,3(93,9-100,6)$ & 7.660 & $222,5(218,1-226,9)$ \\
\hline $32-36$ & 7.403 & $26(25,4-26,6)$ & 3.111 & $11,2(10,8-11,6)$ & 10.514 & $36,9(36,3-37,6)$ \\
\hline $37-41$ & 6.865 & $2,7(2,7-2,8)$ & 3.744 & $1,5(1,4-1,5)$ & 10.609 & $4,2(4,1-4,3)$ \\
\hline 42 ou mais & 131 & $1,7(1,4-2,0)$ & 89 & $1,2(0,9-1,4)$ & 220 & $2,9(2,5-3,6)$ \\
\hline \multicolumn{7}{|l|}{ Tipo de gravidez } \\
\hline Única & 24.740 & $8,5(8,4-8,6)$ & 16.005 & $5,6(5,5-5,7)$ & 40.745 & $14,1(13,9-14,2)$ \\
\hline Múltipla & 1.295 & $20(19,0-21,1)$ & 2.060 & $32,5(31,1-33,9)$ & 3355 & $51,9(50,2-53,6)$ \\
\hline \multicolumn{7}{|l|}{ Tipo de parto } \\
\hline Vaginal & 17.301 & $13,2(13,0-13,4)$ & 9.637 & $7,4(7,3-7,6)$ & 26.938 & $20,5(20,3-20,8)$ \\
\hline Cesáreo & 8.595 & $5,2(5,1-5,3)$ & 8.339 & $5,1(5,0-5,2)$ & 16.934 & $10,2(10,1-10,4)$ \\
\hline
\end{tabular}

IC95\%: intervalo de 95\% de confiança.

* Por 1.000 nascimentos totais;

** Por 1.000 nascidos vivos.

A maior taxa de mortalidade por grupos de causas de óbito foi observada no grupo anteparto $(7,6 \% ; 7,5 \%-7,7 \%$ ) , seguido da prematuridade $(3,6 \%$; 3,6\%o-3,7\%o), das anomalias congênitas $(1,6 \%$; $1,6 \%-1,6 \%$ ), asfixia $(0,8 \% ; 1,1 \%$ - $1,2 \%$ ) e causas especificas $(0,8 \%$; $0,8 \%$ - $0,8 \%$ ).

No grupo anteparto, a maioria dos óbitos $(15.995 ; 74,2 \%)$ apresentou $1.000 \mathrm{~g}$ de peso ou mais (Tabela 2). Desse total, $91,5 \%$ estavam com 28 ou mais semanas de gestação. Dos 6.355 óbitos ocorridos antes do parto e que estavam com $2.500 \mathrm{~g}$ de peso ou mais, a maioria $(4.343 ; 68,3 \%)$ estava entre a 37ạ e 41a semana de gestação.

No grupo das anomalias congênitas, 3.656 (81,9\%), estavam pesando $1.000 \mathrm{~g}$ ou mais (Tabela 2) e $92,9 \%$ estavam com 28 ou mais semanas de gestação. O grupo de causas prematuridade $(3.177 ; 29,9 \%)$, asfixia $(2.593 ; 89,9 \%)$ e causa específica $(2.086 ; 95,6 \%)$ estava com $1.000 \mathrm{~g}$ de peso ou mais (Tabela 2). 


\section{Figura 1}

Distribuição dos óbitos perinatais * segundo classificação de Wigglesworth modificada 20. Brasil, 2018.

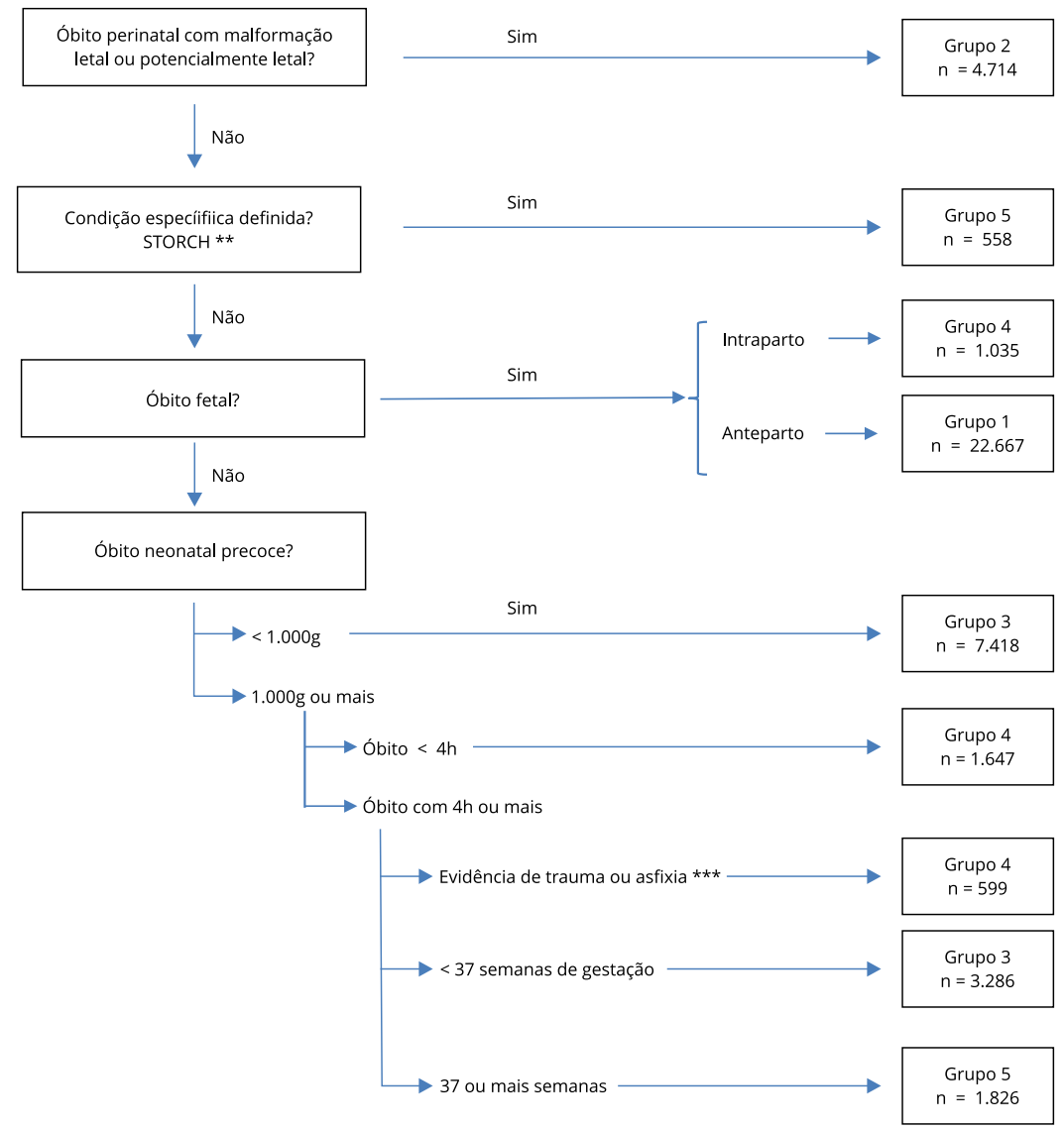

* Excluídos dados ignorados;

** Sífilis, toxoplasmose, rubéola, citomegalovírus, vírus herpes simplex;

*** Classificação Internacional de Doenças - 10ạ revisão: capítulo XVI (P10-P21).

\section{Tabela 2}

Óbitos perinatais por faixa de peso *, segundo classificação de Wigglesworth modificada 20. Brasil, 2018.

\begin{tabular}{|c|c|c|c|c|c|c|c|c|c|c|}
\hline \multirow[t]{3}{*}{ Peso (g) } & \multicolumn{10}{|c|}{ Grupos de causas de óbito } \\
\hline & \multicolumn{2}{|c|}{ Anteparto ** } & \multicolumn{2}{|c|}{$\begin{array}{l}\text { Anomalias } \\
\text { congênitas }\end{array}$} & \multicolumn{2}{|c|}{ Prematuridade } & \multicolumn{2}{|c|}{ Asfixia } & \multicolumn{2}{|c|}{ Causas específicas } \\
\hline & $\mathbf{n}$ & $\%$ & $\mathrm{n}$ & $\%$ & $\mathbf{n}$ & $\%$ & $\mathbf{n}$ & $\%$ & $\mathrm{n}$ & $\%$ \\
\hline$<500$ & - & - & - & - & 1.748 & 16,5 & - & - & 1 & 0,0 \\
\hline $500-999$ & 5.577 & 25,9 & 810 & 18,1 & 5.670 & 53,5 & 291 & 10,1 & 100 & 4,3 \\
\hline $1.000-1.499$ & 3.600 & 16,7 & 800 & 17,9 & 1.486 & 14,0 & 511 & 17,7 & 147 & 6,3 \\
\hline $1.500-2.499$ & 6.040 & 28,0 & 1.514 & 33,9 & 1.273 & 12,0 & 648 & 22,5 & 469 & 20,2 \\
\hline 2.500 ou mais & 6.355 & 29,5 & 1.342 & 30,0 & 418 & 3,9 & 1.434 & 49,7 & 1.603 & 69,1 \\
\hline Total & 21.572 & 100,0 & 4.466 & 100,0 & 10.595 & 100,0 & 2.884 & 100,0 & 2.320 & 100,0 \\
\hline
\end{tabular}

* Excluídos dados ignorados;

** Apenas óbitos fetais. 
Quando a taxa de mortalidade perinatal, por mil nascimentos totais, foi analisada segundo UF, observou-se que, no grupo anteparto, 14 das 27 apresentaram taxas acima da nacional. Destas, oito estiveram na Região Nordeste e quatro na Região Norte. Piauí (10,7\%o), Bahia (10,6\%), Maranhão $(10,6 \%)$ e Amapá $(10,2 \%)$ apresentaram as maiores taxas. A taxa do Piauí foi o dobro da observada no Paraná, a menor (Figura 2a). No grupo de anomalias congênitas, a maior taxa foi em Roraima (2,2\%), seguida de Rondônia, Tocantins, Sergipe e Distrito Federal, com 2,1\% cada (Figura 2b). Com relação à prematuridade, destacaram-se Amapá $(7,1 \%)$, Sergipe $(5,8 \%)$ e Bahia $(4,9 \%)$ (Figura $2 \mathrm{c})$. No grupo de asfixia, o Acre (2,4\%) apresentou uma taxa quatro vezes maior que o Distrito Federal $(0,6 \%)$, Bahia $(1,9 \%)$ e Amazonas (1,9\%) (Figura 2d). Nas causas específicas, destacaram-se Roraima (2,7\%), Acre $(1,8 \%)$ e Rio de Janeiro (1,7\%) (Figura 2e).

\section{Discussão}

Os resultados apontam que no Brasil, em 2018, a taxa de mortalidade perinatal foi de 15,5\% nascimentos totais e os óbitos neonatais precoces representaram a maioria dos óbitos infantis, menos, no entanto, da metade dos óbitos perinatais. A taxa de mortalidade no grupo anteparto foi o dobro da observada no grupo de prematuridade e quase cinco vezes maior que a registrada nas anomalias congênitas.

A maioria dos óbitos ocorridos no período anteparto foi de fetos com $1.000 \mathrm{~g}$ ou mais de peso e, dentre estes, a quase totalidade apresentou mais de 28 semanas de gestação. A mortalidade perinatal vem sendo discutida há bastante tempo como um indicador de resultado dos cuidados pré-natais e das condições maternas durante a gestação 7,25. Por isso, faz-se necessário concentrar esforços na díade mãe-bebê ao investir em cuidados pré-natais para reduzir a mortalidade perinatal, visto que melhoras nos padrões de atendimento e do acesso a esses cuidados podem reduzir a mortalidade.

O grupo das anomalias congênitas apresentou a terceira maior taxa de mortalidade, segundo a classificação adotada. A seleção deste grupo foi feita considerando-se as malformações letais ou potencialmente letais descritas na literatura. Existem várias maneiras diferentes de interpretar, teoricamente, uma condição perinatal como sendo letal. Porém, raras anomalias mais comumente descritas como letais são realmente letais no sentido estrito. A sobrevida prolongada foi descrita em todas as condições listadas, com exceção da agenesia renal bilateral. Todavia, mesmo nessa condição, a sobrevivência seria concebível com suporte intensivo 21. Por isso, é primordial assegurar o acesso ao diagnóstico, ainda no período gestacional, bem como a terapia para as lesões potencialmente tratáveis.

A prematuridade configura-se como o grupo composto por recém-nascidos com idade gestacional menor que 37 semanas e aqueles com peso inferior a 1.000g. As maiores taxas de mortalidade neonatal precoce foram registradas nos grupos de extremo baixo peso $(<1.000 \mathrm{~g})$ e até 27 semanas de gestação (pré-termo extremo). Estudos mostram a gravidez gemelar, antecedente de parto prematuro, a presença de sangramento, pressão arterial alta, infecção do trato genital, diabetes, pré-eclampsia/ eclampsia, pré-natal inadequado e ausente como fatores de risco para a ocorrência de prematuridade 26,27. Esses achados apontam que, para evitar a ocorrência desses óbitos, é preciso centrar esforços em dois momentos distintos: nos cuidados com a mulher por meio dos manejos pré-natal e obstétricos adequados e, posteriormente ao nascimento, no atendimento neonatal. Com o progresso contínuo nos cuidados perinatais, o limite da viabilidade humana mudou para uma idade gestacional cada vez mais precoce 28 , porém, a taxa de mortalidade nos nascidos extremamente prematuros ainda permanece alta. Por isso, faz-se necessário investir nos cuidados de saúde perinatais apropriados para o tratamento desse grupo.

No grupo asfixia, a quase totalidade dos óbitos estava com 1.000g ou mais de peso e, dentre esses, a maioria estava com $2.500 \mathrm{~g}$ ou mais. A asfixia perinatal é reconhecida como uma medida sensível da qualidade da assistência prestada tanto à gestante quanto ao recém-nascido, visto que ela surge em decorrência da falta de oxigenação adequada fetal-neonatal no periparto, ao nascimento e nos primeiros minutos de vida 29. Desta forma, os óbitos por essa causa apresentam-se como potencialmente evitáveis por meio da assistência adequada, que assegure um diagnóstico e tratamento precoces no pré-natal, parto e ao nascimento. 


\section{Figura 1}

Distribuição espacial da taxa de mortalidade perinatal por grupos de causas e Unidade da Federação, segundo classificação de Wigglesworth modificada 20. Brasil, 2018.

2a) Anteparto

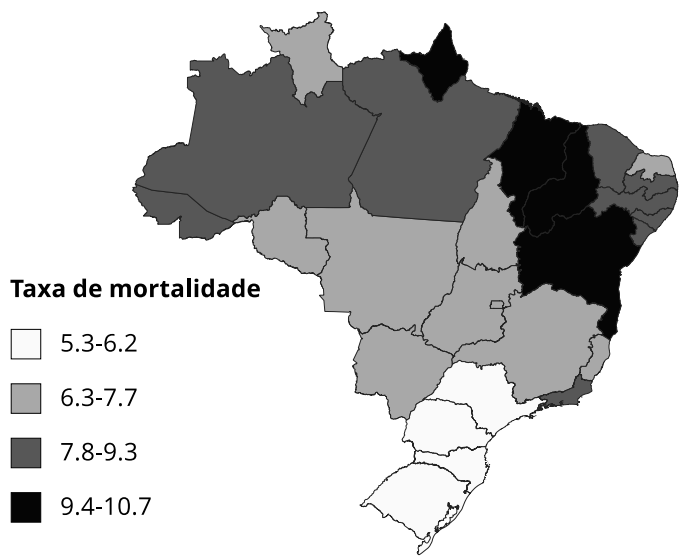

2c) Prematuridade

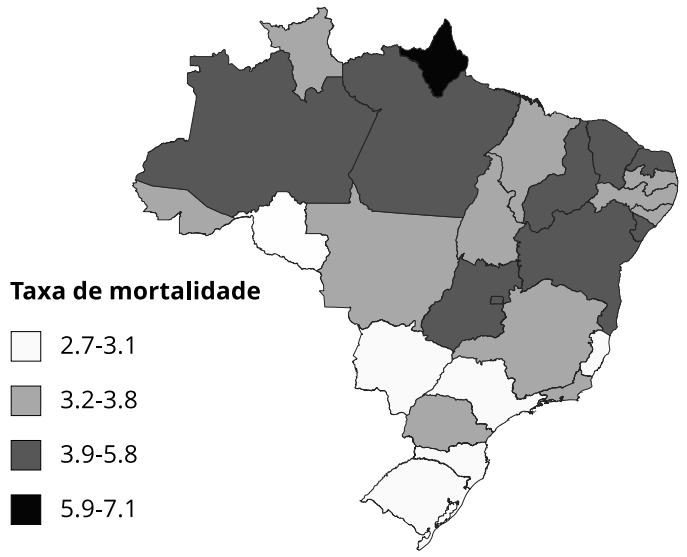

2e) Causas específicas

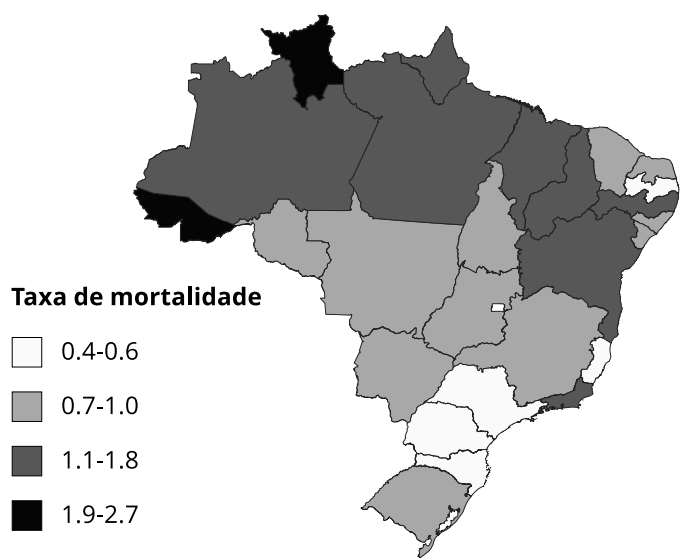

2b) Malformação congênita

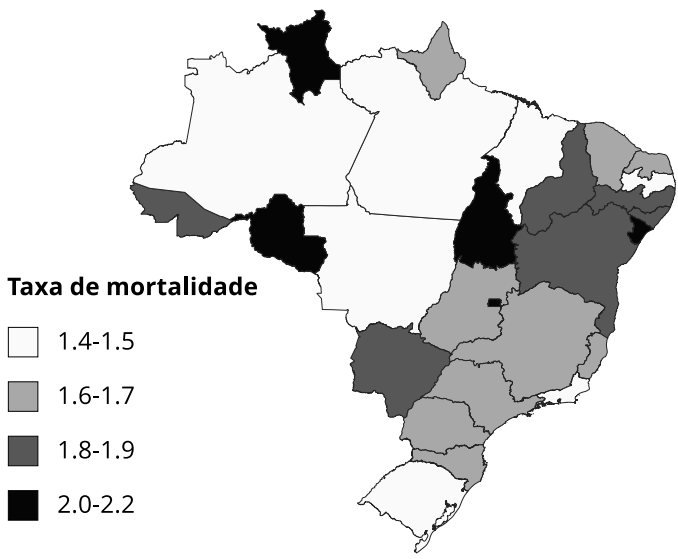

2d) Asfixia

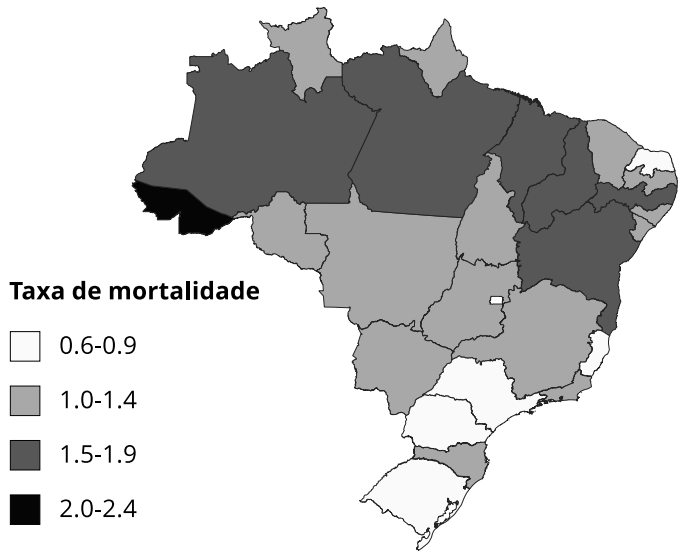


A quase totalidade dos óbitos registrados no grupo de causas específicas foi devido à sífilis. Tratase de uma infecção sexualmente transmissível, cujo tratamento com antibiótico, quando ofertado em tempo oportuno, pode assegurar a remissão completa da doença na mãe e impedir a transmissão vertical para o bebê, tornando, dessa forma, evitáveis os óbitos por essa causa 30. Desde 2005, os manuais de atenção qualificada e humanizada do Ministério da Saúde têm como critério fundamental para o acompanhamento do pré-natal a solicitação de vários exames, dentre eles, a sorologia para sífilis. Com diagnóstico confirmado, deve-se instituir o tratamento e o acompanhamento da gestante 31 . Todavia, um estudo mostrou que, entre 2010 e 2015, ocorreu aumento nas taxas de incidência de sífilis congênita em crianças menores de um ano de idade e nas taxas de mortalidade infantil, natimortos e abortos espontâneos por sífilis. Esse estudo também apontou a correlação entre taxas de natimortos causadas por sífilis e assistência pré-natal inadequada 32.

A análise da taxa de mortalidade, segundo o grupo de causas, apontou grandes discrepâncias dos resultados entre as UFs. Essas diferenças podem refletir tanto as dificuldades no acesso aos serviços de saúde como as desigualdades socioeconômicas. Por exemplo, as menores taxas de mortalidade por anomalias congênitas registradas em alguns estados da Região Norte podem dever-se ao subdiagnóstico, causado provavelmente pela dificuldade de acesso a exames durante a gestação e após o parto. Outrossim, as anomalias costumam desapontar como causas importantes de óbito em lugares onde as demais causas de mortalidade infantil já foram controladas 33,34,35. Outro ponto a ser destacado é que o Norte e o Nordeste, regiões com as maiores taxas de mortalidade perinatal em todos os grupos, apresentam os mais baixos indicadores econômicos e de vulnerabilidade social do país 36 . O fato de a maior taxa de mortalidade perinatal ser observada entre mulheres com baixa escolaridade fortalece essa hipótese.

Os dados analisados nesse estudo são de fontes secundárias e, por isso, impõem algumas limitações aos resultados. Estudos que avaliaram os dados do óbito fetal e não fetal no SIM mostraram que a qualidade variou de boa a excelente e vem aumentando ao longo do tempo 37,38. Assim, acredita-se que os valores ignorados excluídos das análises não alteraram, substancialmente, os resultados desse trabalho. Por outro lado, de acordo com estimativas indiretas do Instituto Brasileiro de Geografia e Estatística (IBGE) para o Brasil, em 2008, o SIM e o SINASC, ainda apresentam uma sub-enumeração para menores de um ano, limitando, dessa forma, o uso do cálculo direto para estimar a mortalidade. As menores coberturas ocorrem nas regiões Norte e Nordeste e alguns estados do Centro-oeste, seguindo o padrão de desigualdade socioeconômica ${ }^{39}$. Sendo assim, os indicadores nessas regiões podem estar ainda piores, se considerada a provável subestimação dos dados.

A classificação de Wigglesworth modificada baseia-se em uma árvore de decisão, que vai gerando grupos específicos e excludentes ao longo das análises 20. Porém, os indivíduos que evoluíram a óbito, na sua complexidade, podem apresentar mais de uma afecção contribuinte, mas são classificados em apenas uma delas, o que indica que o valor da taxa é da afecção e não dos indivíduos. Mesmo assim, os resultados encontrados com a utilização desse método de análise são relevantes para apontar áreas prioritárias de cuidados de saúde e prevenção em saúde infantil.

O investimento em cuidados pré-natais e ao nascimento pode ter um impacto triplo na redução da mortalidade. Segundo a OMS, a alta cobertura de cuidados na época do nascimento e os cuidados com recém-nascidos pequenos e doentes salvariam quase três milhões de vidas (mulheres, recém-nascidos e fetos) a cada ano 10. Por isso, o tema da mortalidade perinatal precisa assumir um lugar proeminente na agenda atual das ações de saúde no Brasil, por meio do fortalecimento e aprimoramento das iniciativas existentes no país 40,41. Só assim será possível reduzir a ocorrência de tantos óbitos perinatais e alcançar a meta pactuada nos Objetivos de Desenvolvimento Sustentável tanto para mortalidade neonatal como para mortalidade materna 42,43 .

Por fim, a classificação dos óbitos perinatais usada nesse trabalho forneceu informações relevantes para o planejamento de intervenções nos cuidados com a gestante e os recém-nascidos. Os resultados podem ajudar a definir as áreas a serem priorizadas, visando à redução da mortalidade perinatal e, de forma indireta, da materna. 


\section{Colaboradores}

A. A. Nobrega contribuiu no desenho e elaboração do estudo, análise e interpretação dos dados e redação. Y. M. M. B. Mendes, M. J. Miranda e A. C. C. Santos contribuíram na elaboração e revisão do artigo. A. P. Lobo e D. L. Porto contribuíram na revisão do artigo. G. V. A. França contribuiu na análise crítica e revisão do artigo. Todos os autores aprovaram a versão final do artigo.

\section{Informações adicionais}

ORCID: Aglaer Alves da Nobrega (0000-0003-29395002); Yluska Myrna Meneses Brandão e Mendes (0000-0002-6165-6930); Marina Jorge de Miranda (0000-0002-0056-7731); Augusto César Cardoso dos Santos (0000-0002-1499-9105); Andréa de Paula Lobo (0000-0002-7801-9866); Denise Lopes Porto (0000-0002-1995-4715); Giovanny Vinícius Araújo de França (0000-0002-7530-2017).

\section{Referências}

1. Organização Mundial da Saúde. Classificação Estatística Internacional de Doenças e Problemas Relacionados à Saúde - 10a revisão. São Paulo: Edusp; 2009.

2. Saleem S, McClure EM, Goudar SS, Patel A, Esamai F, Garces A, et al. A prospective study of maternal, fetal and neonatal deaths in lowand middle-income countries. Bull World Health Organ 2014; 92:605-12.

3. Sharma S, Sidhu H, Kaur S. Analytical study of intrauterine fetal death cases and associated maternal conditions. Int J Appl Basic Med Res 2016; 6:11-3.

4. Barbeiro FMS, Fonseca SC, Tauffer MG, Ferreira MSS, Silva FP, Ventura PM, et al. Óbitos fetais no Brasil: revisão sistemática. Rev Saúde Pública 2015; 49:22.

5. Kale PL, Mello Jorge MHP, Fonseca SC, Cascão AM, Silva KS, Reis AC, et al. Mortes de mulheres internadas para parto e por aborto e de seus conceptos em maternidades públicas. Ciênc Saúde Colet 2018; 23:1577-90.

6. Fonseca SC, Coutinho ESF. Características biológicas e evitabilidade de óbitos perinatais em uma localidade na cidade do Rio de Janeiro, 1999 a 2003. Rev Bras Saúde Matern Infant 2008; 8:171-8.

7. Richardus JH, Graafmans WC, Verloove-Vanhorick SP, Mackenbach JP. The perinatal mortality rate as an indicator of quality of care in international comparisons. Med Care 1998; 36:54-66.

8. Rêgo MGS, Vilela MBR, Oliveira CM, Bonfim CV. Óbitos perinatais evitáveis por intervenções do Sistema Único de Saúde do Brasil. Rev Gaúch Enferm 2018; 39:e20170084.

9. Blencowe H, Cousens S, Jassir FB, Say L, Chou D, Mathers C, et al. National, regional, and worldwide estimates of stillbirth rates in 2015 , with trends from 2000: a systematic analysis. Lancet Glob Health 2016; 4:e98-108.

10. World Health Organization. Every newborn: an action plan to end preventable deaths. Geneva: World Health Organization; 2014.

11. Menezes AMB, Barros FC, Horta BL, Matijasevich $A$, Bertoldi $A D$, Oliveira $P D$, et al. Stillbirth, newborn and infant mortality: trends and inequalities in four population-based birth cohorts in Pelotas, Brazil, 1982-2015. Int J Epidemiol 2019; 48 Suppl 1:i54-62.

12. Barros PS, Aquino ÉC, Souza MR. Fetal mortality and the challenges for women's health care in Brazil. Rev Saúde Pública 2019; 53:12.

13. Lanskya S, França E, Leal MC. Mortalidade perinatal e evitabilidade: revisão da literatura. Rev Saúde Pública 2002; 36:759-72.

14. Secretaria de Vigilância em Saúde; Secretaria de Atenção à Saúde, Ministério da Saúde. Manual de vigilância do óbito infantil e fetal e do Comitê de Prevenção do Óbito Infantil e Fetal. Brasília: Ministério da Saúde; 2009. 
15. Secretaria de Vigilância em Saúde, Ministério da Saúde. Portaria no 116, de 11 de fevereiro de 2009. Regulamenta a coleta de dados, fluxo e periodicidade de envio das informações sobre óbitos e nascidos vivos para os Sistemas de Informações em Saúde sob gestão da Secretaria de Vigilância em Saúde. Diário Oficial da União 2009; 12 fev.

16. Departamento de Análise de Saúde e Doenças Não Transmissíveis, Secretaria de Vigilância em Saúde, Ministério da Saúde. Indicadores de cobertura que utilizam a metodologia do Busca Ativa. http://svs.aids.gov.br/dantps/acessoa-informacao/acoes-e-programas/busca-ati $\mathrm{va} /$ indicadores-de-saude/cobertura/ (acessado em 10/Jul/2020).

17. Jorge MH, Gotlieb SL, Soboll ML, Almeida MF, Latorre MR. Avaliação do Sistema de Informação sobre Nascidos Vivos e o uso de seus dados em epidemiologia e estatísticas de saúde. Rev Saúde Pública 1993; 27 Suppl:1-44.

18. Rede Interagencial de Informação para a Saúde. Indicadores de saúde no Brasil: conceitos e aplicações. 2a Ed. Brasília: Organização Pan-Americana da Saúde; 2008.

19. Wigglesworth JS. Monitoring perinatal mortality: a pathophysiological approach. Lancet 1980; 316:684-6.

20. Keeling JW, MacGillivray I, Golding J, Wigglesworth J, Berry J, Dunn PM. Classification of perinatal death. Arch Dis Child 1989; 64(10 Spec No):1345-51.

21. Wilkinson D, Thiele P, Watkins A, De Crespigny L. Fatally flawed? A review and ethical analysis of lethal congenital malformations. BJOG 2012; 119:1302-8.

22. Ministério da Saúde. Portaria no 72, de 11 de janeiro de 2010. Estabelece que a vigilância do óbito infantil e fetal é obrigatória nos serviços de saúde (públicos e privados) que integram o Sistema Único de Saúde (SUS). Diário Oficial da União 2010; 12 jan.

23. Brasil. Decreto no 7.724 , de 16 de maio de 2012. Regulamenta a Lei no 12.527 , de 18 de novembro de 2011, que dispõe sobre o acesso a informações previsto no inciso XXXIII do caput do art. 5o , no inciso II do $\$ 3$ o do art. 37 e no $\$ 2$ o do art. 216 da Constituição. Diário Oficial da União 2012; 16 mai.

24. Ministério da Saúde. Resolução no 510, de 7 de abril de 2016. Diário Oficial da União 2016; 24 mai.

25. Kaunitz AM, Spence C, Danielson TS, Rochat RW, Grimes DA. Perinatal and maternal mortality in a religious group avoiding obstetric care. Am J Obstet Gynecol 1984; 150:826-31.

26. Ahumada-Barrios ME, Alvarado GF. Fatores de risco para parto prematuro em um hospital. Rev Latinoam Enferm 2016; 24:e2750.

27. Oliveira AA, Almeida MF, Silva ZP, Assuncao PL, Silva AMR, Santos HG, et al. Factors associated with preterm birth: from logistic regression to structural equation modeling. Cad Saúde Pública 2020; 35:e0211917.
28. Pignotti MS, Donzelli G. Perinatal care at the threshold of viability: an international comparison of practical guidelines for the treatment of extremely preterm births. Pediatrics 2008; 121:e193-8.

29. Daripa M, Caldas HMG, Flores LPO, Waldvogel BC, Guinsburg R, Almeida MFB. Asfixia perinatal associada à mortalidade neonatal precoce: estudo populacional dos óbitos evitáveis. Rev Paul Pediatr 2013; 31:37-45.

30. Saloojee H, Velaphi S, Goga Y, Afadapa N, Steen R, Lincetto O. The prevention and management of congenital syphilis: an overview and recommendations. Bull World Health Organ 2004; 82:424-30.

31. Programa Nacional de DST e Aids, Secretaria de Vigilância em Saúde, Ministério da Saúde. Diretrizes para o controle da sífilis congênita. Brasília: Ministério da Saúde; 2005. (Série Manuais, 62).

32. Bezerra MLMB, Fernandes FECV, Nunes JPO, Baltar SLSMA, Randau KP. Congenital syphilis as a measure of maternal and child healthcare, Brazil. Emerg Infect Dis 2019; 25:1469-76.

33. Secretaria de Vigilância em Saúde, Ministério da Saúde. Anomalias congênitas no Brasil, 2010 a 2018: análise dos dados de sistemas de informação para o fortalecimento da vigilância e atenção em saúde. https://www.saude.gov. $\mathrm{br} / \mathrm{images} / \mathrm{pdf} / 2020 / \mathrm{marco} / 27 /$ Boletim-epi demiologico-SVS-13.pdf (acessado em 10/ $\mathrm{Jul} / 2020)$.

34. Victora CG, Aquino EM, Do Carmo Leal M, Monteiro CA, Barros FC, Szwarcwald CL. Maternal and child health in Brazil: progress and challenges. Lancet 2011; 377:1863-76.

35. Cardoso-dos-Santos AC, Medeiros-de-Souza AC, Bremm JM, Alves RFS, Araújo VEM, Leite JCL, et al. List of priority congenital anomalies for surveillance under the Brazilian Information System on Live Births. Epidemiol Serv Saúde 2021; 30:e2020835.

36. Instituto de Pesquisa Econômica Aplicada. A nova plataforma da vulnerabilidade social: primeiros resultados do índice de vulnerabilidade social para a série histórica da PNAD (20112015) e desagregações por sexo, cor e situação de domicílios. Rio de Janeiro: Instituto de Pesquisa Econômica Aplicada; 2018.

37. Nóbrega AA, Souza ACM, Marques LJP, Frutuoso LCV, Almeida MF, Miranda MJ, et al. Avaliação da qualidade dos dados do óbito fetal no Sistema de Informação sobre Mortalidade do Brasil. In: Departamento de Vigilância de Doenças e Agravos não Transmissíveis e Promoção da Saúde, Secretaria de Vigilância em Saúde, Ministério da Saúde, editor. Saúde Brasil 2018 - uma análise de situação de saúde e das doenças e agravos crônicos: desafios e perspectivas. Brasília: Ministério da Saúde; 2018. p. 359-75. 
38. Souza A, Rabello Neto D. Avaliação da qualidade dos dados sobre mortalidade no Brasil de 2000 a 2016. In: Departamento de Vigilância de Doenças e Agravos não Transmissíveis e Promoção da Saúde, Secretaria de Vigilância em Saúde, Ministério da Saúde, editor. Saúde Brasil 2018 - uma análise de situação de saúde e das doenças e agravos crônicos: desafios e perspectivas. Brasília: Ministério da Saúde; 2018. p. 377-92.

39. Frias PG, Szwarcwald CL, Lira PIC. Estimação da mortalidade infantil no contexto de descentralização do Sistema Único de Saúde (SUS). Rev Bras Saúde Matern Infant 2011; 11:463-70.

40. Departamento de Ciência e Tecnologia, Secretaria de Ciência, Tecnologia e Insumos Estratégicos, Ministério da Saúde. Síntese de evidências para politicas de saúde: reduzindo a mortalidade perinatal. 2a Ed. Brasília: Ministério da Saúde; 2016.
41. Justino DCP, Lopes MS, Santos CDP, Andrade FB. Avaliação histórica das políticas públicas de saúde infantil no Brasil: revisão integrativa. Rev Ciênc Plur 2019; 5:71-88.

42. United Nations. SDG Indicators - metadata repository. https://unstats.un.org/sdgs/meta data/ (acessado em 23/Mar/2021).

43. Instituto de Pesquisa Econômica Aplicada. Cadernos ODS 3: assegurar uma vida saudável e promover o bem-estar para todas e todos, em todas as idades. O que mostra o retrato do Brasil? Rio de Janeiro: Instituto de Pesquisa Econômica Aplicada; 2019. 


\begin{abstract}
Perinatal mortality includes fetal mortality and early neonatal mortality ( 0 to 6 days of life). The study described perinatal deaths in Brazil in 2018 according to the modified Wigglesworth classification. The data sources were the Brazilian Mortality Information System and the Brazilian Information System on Live Births. Fetal mortality and perinatal mortality rates were calculated per 1,000 total births (live births plus stillbirths) and the early neonatal mortality rate per 1,000 live births, compared using their respective $95 \%$ confidence intervals (95\% CI). Perinatal deaths were classified in groups of antepartum causes, congenital anomalies, prematurity, asphyxia, and specific causes. For each group of causes, the study calculated the number of deaths by weight group, in addition to mortality rates and respective $95 \% \mathrm{CI}$, besides the spatial distribution of mortality rates by state of Brazil. A total of 35,857 infant deaths were recorded, of which 18,866 (52.6\%) were early neonatal deaths, while stillbirths totaled 27,009. Perinatal deaths totaled 45,875, for a mortality rate of $15.5 \%$ births. The highest mortality rate (7.6\%; $7.5 \%$ - $7.7 \%$ ) was observed in the antepartum group, followed by prematurity (3.6\%; 3.6\%-3.7\%). In the antepartum group, 14 of the 27 states (eight of which in the Northeast and four in the North) presented perinatal mortality rates above the national rate. Perinatal mortality in Brazil was high, and most deaths could have been prevented with investment in prenatal and childbirth care.
\end{abstract}

Perinatal Mortality; Fetal Death; Neonatal Death

\section{Resumen}

La mortalidad perinatal engloba la mortalidad fetal y neonatal precoz ( 0 a 6 días). Este estudio describió los óbitos perinatales ocurridos en Brasil en 2018, según la clasificación de Wigglesworth modificada. Las fuentes de datos fueron los Sistemas de Información sobre Mortalidad y sobre Nacidos Vivos. Se calcularon las tasas de mortalidad fetal y perinatal por 1.000 nacimientos totales (nacidos vivos más mortinatos) y la tasa de mortalidad neonatal precoz por 1.000 nacidos vivos, y se compararon usando sus respectivos intervalos de 95\% de confianza (IC95\%). Los óbitos perinatales se clasificaron en los grupos de causas: anteparto, anomalías congénitas, prematuridad, asfixia y causas específicas. Se calculó, para cada grupo de causas, el número de óbitos por franja de peso, además de las tasas de mortalidad y los respectivos IC95\%, y se realizó la distribución espacial de las tasas de mortalidad por Unidad de la Federación (UF). Se registraron 35.857 óbitos infantiles, siendo 18.866 (52,6\%) neonatales precoces; los mortinatos sumaron 27.009. Los óbitos perinatales totalizaron 45.875, ascendiendo a una tasa de mortalidad de un $15,5 \%$ nacimientos. La mayor tasa de mortalidad (7,6\%; 7,5\%o-7,7\%) se observó en el grupo anteparto, seguido de la prematuridad $(3,6 \% ; 3,6 \%-3,7 \%)$. En el grupo anteparto, 14 de las 27 UFs (estando ocho en la región Nordeste y cuatro en la región Norte) presentaron tasas de mortalidad perinatal por encima de la nacional. La tasa de mortalidad perinatal en Brasil se mostró elevada y la mayoría de los óbitos podría ser prevenido con inversión en cuidados prenatales y en el nacimiento.

Mortalidad Perinatal; Muerte Fetal; Muerte

Neonatal
Recebido em 13/Jan/2021

Versão final reapresentada em 06/Jul/2021

Aprovado em 15/Jul/2021 